\title{
Reduction of Benzoquinones to Hydroquinones via Spontaneous Reaction with Glutathione and Enzymatic Reaction by S- Glutathionyl-Hydroquinone Reductases
}

\author{
L. K. Metthew Lam, ${ }^{\dagger}$ Zhicheng Zhang, ${ }^{\ddagger}$ Philip G. Board, ${ }^{\S}$ and Luying Xun ${ }^{* \dagger}$ \\ ${ }^{\dagger}$ School of Molecular Biosciences, and ${ }^{\ddagger}$ Department of Chemistry, Washington State University, Pullman, Washington, United States \\ $\S$ John Curtin School of Medical Research, Australian National University, Canberra, Australian Capital Territory, Australia
}

\begin{abstract}
S-Glutathionyl-hydroquinone reductases (GSHQRs) are a new class of glutathione transferases, widely present in bacteria, halobacteria, fungi, and plants. They catalyze glutathione (GSH)-dependent reduction of GStrichloro- $p$-hydroquinone to trichloro- $p$-hydroquinone. Since GS-trichloro- $p$-hydroquinone is uncommon in nature, the extensive presence of GS-HQRs suggests they use common GS-hydroquinones. Here we demonstrate that several benzoquinones spontaneously reacted with GSH to form

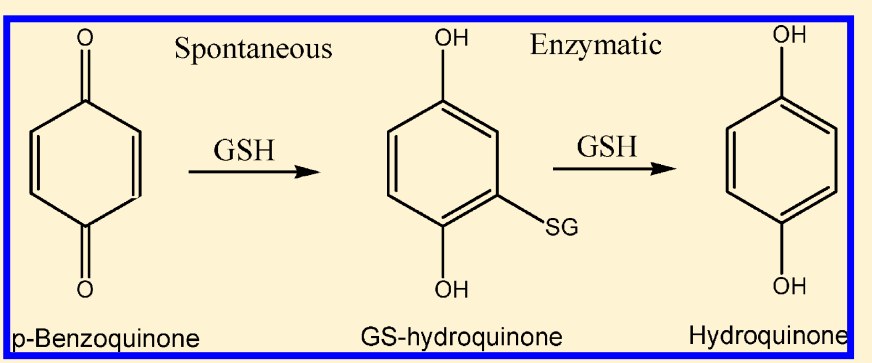
GS-hydroquinones via Michael addition, and four GS-HQRs

from yeast and bacteria reduced the GS-hydroquinones to the corresponding hydroquinones. The spontaneous and enzymatic reactions led to the reduction of benzoquinones to hydroquinones with the concomitant oxidation of GSH to oxidized glutathione (GS-SG). The enzymes did not use GS-benzoquinones or other thiol-hydroquinones, for example, S-cysteinylhydroquinone, as substrates. Apparent kinetic parameters showed the enzymes preferred hydrophobic, bulky substrates, such as GS-menadiol. The broad substrate range and their wide distribution suggest two potential physiological roles: channeling GShydroquinones back to hydroquinones and reducing benzoquinones via spontaneous formation of GS-hydroquinones and then enzymatic reduction to hydroquinones. The functions are likely important in metabolic pathways with quinone intermediates.
\end{abstract}

$G$ lutathionyl-(chloro)hydroquinone reductases (GS-

HQRs) are common in bacteria, halobacteria, fungi, and plants. ${ }^{1}$ They are related to several classes of newly discovered glutathione transferases (GST) within the cytosolic GST superfamily. Unlike other classes of the GST superfamily, the new classes have little glutathione (GSH) conjugating activity but catalyze GSH-dependent reductions. The Lambda-class GSTs perform only GSH-dependent thioltransferase reactions, and the dehydroascorbate reductases catalyze both dehydroascorbate reduction and thioltransferase reactions. ${ }^{2}$ The Omegaclass GSTs and GS-HQRs are more similar, catalyzing GSHdependent thioltransferase reactions as well as the reduction of dehydroascorbate and dimethylarsinate. ${ }^{1,3,4}$ However, although the Omega-class GSTs reduce substrates such as $S$-(phenacyl) glutathione, ${ }^{5}$ they do not reduce GS-trichloro- $p$-hydroquinone (GS-TriCH). ${ }^{1}$ In contrast, GS-HQRs can reduce GS-TriCH to $\mathrm{TriCH}$ at the expense of GSH. Members of the two classes share less than $20 \%$ sequence identity. ${ }^{1}$ Structural characterization of a GS-HQR (PcGHR1) of white rot fungus Phanerochaete chrysosporium has revealed structural differences of GS-HQRs from other GSTs. ${ }^{3}$ The enzyme has a new homodimerization mode, and its catalytic site is buried by a long N-terminal coil instead of open. Thus, GS-HQRs represent a new class of GSTs with special activities and unique structural properties. Meux et al. have proposed a new GST class xi for GS-HQRs. ${ }^{3}$
PcpF is the first characterized GS-HQR, and it plays a maintenance role in pentachlorophenol (PCP) degradation by Sphingobium chlorophenolicum ATCC 29327. ${ }^{6}$ Tetrachloro- $p$ hydroquinone is a metabolic intermediate, and PcpC, a GST, converts it to $\mathrm{TriCH}$ and then dichloro- $p$-hydroquinone $(\mathrm{DiCH})$ with concomitant oxidation of GSH to oxidized glutathione (GS-SG). ${ }^{7,8}$ When a Cys residue at the active site of $\mathrm{PcpC}$ is oxidized, the oxidatively damaged PcpC produces GSTriCH and GS-DiCH conjugates, ${ }^{9}$ which are not substrates for PcpC. PcpF channels the conjugates back to TriCH and $\mathrm{DiCH}$, respectively, with the coconsumption of GSH. When $p c p F$ is inactivated, the $S$. chlorophenolicum mutant degrades PCP more slowly than the wild type does. ${ }^{6}$ GS-TriCH and GS-DiCH are expected to accumulate and to be toxic to the $p c p F$ mutant.

The chlorohydroquinones are metabolic intermediates of PCP, which was first introduced into the environment as a wood preservative in the 1930 s. $^{10}$ Because several tested GSHQRs all reduced GS-TriCH, they are expected to use naturally occurring GS-hydroquinones. ${ }^{1,11}$ Benzoquinones spontaneously react with GSH via Michael addition to form GS-hydroquinones (reduced form) (Figure 1, step 2). ${ }^{12}$ In addition, benzoquinones and GS-benzoquinones can be reduced by $\mathrm{NAD}(\mathrm{P}) \mathrm{H}$-dependent enzymes (Figure 1 step 1 reverse). ${ }^{13}$ Since benzoquinones can damage biological

Received: April 13, 2012

Published: June 11, 2012 


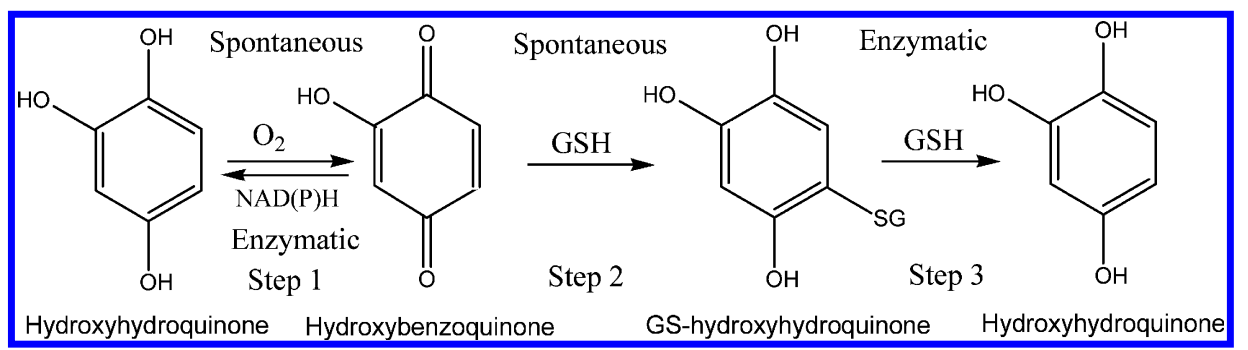

Figure 1. The cycle of HHQ oxidation, conjugation, and reduction. Step 1 forward reaction is spontaneous and reverse reaction is catalyzed by quinone reductases. Step 2 is spontaneous via Michael addition. Step 3 is proposed to be catalyzed by GS-HQRs.

molecules by forming covalent bonds at nucleophilic sites via Michael addition, ${ }^{14}$ their spontaneous reaction with GSH and their reduction by enzymes are likely detoxifying mechanisms. Hydroquinones are subject to autoxidation (Figure 1 step 1), which is a complex process with the formation of semiquinones, superoxide, and hydrogen peroxide. ${ }^{12}$ Because GS-hydroquinones are more prone to autoxidation than the corresponding hydroquinones, ${ }^{15}$ the conjugates are expected to generate more reactive oxygen species (ROS) and be more harmful.

Literature on the formation and toxicity of GS-hydroquinones is extensive. Rat kidney necrosis that is observed after administration of bromobenzene, $o$-bromophenol, or 2-bromo$p$-hydroquinone is attributed to 2-bromo- $S$-(diglutathionyl)hydroquinone, which is formed in the liver and subsequently transported to the kidney. ${ }^{16}$ Toxicity of 2-bromo-S-(diglutathionyl)-hydroquinone is linked to its ability to generate ROS that cause DNA damage. ${ }^{17}$ Hydroquinone is transformed to mono-, di-, tri-GS-hydroquinones in rats, ${ }^{12}$ and the conjugates have been linked to kidney damage. ${ }^{18}$ Hydroquinone is a metabolite of benzene and phenol and is present in the smoke of cigarettes. ${ }^{19}$ Nonenzymatic formation of GS-menadione (3glutathionyl-2-methyl-1,4-naphthoquinone or thiodione) from menadione (vitamin $\mathrm{K} 3$ ) and GSH is accompanied by ROS formation in perfused rat liver. ${ }^{20}$ Thus, GS-hydroquinones may be responsible elevated ROS formation in vivo.

Mammals do not have GS-HQRs; ${ }^{1,21}$ however, GS-hydroquinones are pumped out of cells and carried in blood to kidney, where they are converted to $\mathrm{N}$-acetyl-cysteinyl-Sconjugates (mercapturic acids) and released in the urine. GSHQRs are common in bacteria, halobacteria, fungi, and plants, suggesting that they may convert GS-hydroquinones back to hydroquinones (Figure 1, step 3), which can be further metabolized, thus minimizing the toxicity of quinones and quinone-conjugates. A GS-HQR in P. chrysosporium (PcGHR1) is reported to reduce both GS-hydroquinone (GS-HQ) and GS-menadione with the coconsumption of GSH. ${ }^{3}$ In this study, we characterized the formation of several GS-hydroquinones, proved the enzymatic reduction of the conjugates by three bacterial GS-HQRs and one yeast GS-HQR, and determined apparent kinetic parameters. The results point to the general ability of GS-HQRs to reduce a broad range of GShydroquinones, providing clues about the physiological role of GS-HQRs.

\section{EXPERIMENTAL PROCEDURES}

Chemicals. All chemicals were obtained from Aldrich Chemical Co. (Milwaukee, WI), Fisher Scientific Co. (Pittsburgh, PA), or Sigma Chemical Co. (St. Louis, MO).

GS-HQRs and hGST01-1. Recombinant PcpF, EcYqjG of Escherichia coli, CnYqjG of Cupriavidus necator (formerly Ralstonia eutropha) JMP134, ECM4 of Saccharomyces cerevisiae
(Baker's yeast) and hGSTO1-1 (human omega class GST 1) were produced in Escherichia coli strain BL21(DE3) as fusion proteins with a C-terminal $\mathrm{His}_{6}$-Tag and purified with $\mathrm{Ni}^{2+}$ nitrilotriacetate-agarose resin as previously reported. ${ }^{1}$ The purified proteins were stable for several months when stored in $40 \mathrm{mM}$ potassium phosphate buffer $(\mathrm{pH}$ 7) with $1 \mathrm{mM}$ dithiothreitol and $10 \%$ glycerol at $-80{ }^{\circ} \mathrm{C}$.

GS-Hydroquinone Preparation. Stock solutions of benzoquinones and hydroquinones were prepared in ethanol at $100 \mathrm{mM}$, except menadione at $25 \mathrm{mM}$ because of its low solubility. GSH stock was $200 \mathrm{mM}$ in distilled water. GShydroxy-p-hydroquinone (GS-HHQ) was prepared with HHQ as the starting material. HHQ $(100-500 \mu \mathrm{M})$ was autoxidized to hydroxy- $p$-benzoquinone (HBQ) in $40 \mathrm{mM}$ Tris buffer $(\mathrm{pH}$ 8 ) at $23{ }^{\circ} \mathrm{C}$ in about 15 min. ${ }^{22}$ Excess $(1 \mathrm{mM}) \mathrm{GSH}$ was then added to produce GS-HHQ which occurred spontaneously and instantaneously. Immediately, $\mathrm{NaBH}_{4}$ was added to $1 \mathrm{mM}$ to prevent autoxidation of GS-HHQ. When equal volumes of 200 $\mathrm{mM}$ GSH and $25 \mathrm{mM}$ menadione stock solutions were mixed, the reaction took about $30 \mathrm{~min}$ to reach more than $95 \%$ completion. The produced GS-menadiol was not stable and was autoxidized to GS-menadione in the reaction mixture. The mixture was then kept on ice and was used within $60 \mathrm{~min}$. GSmenadione was added to $40 \mathrm{mM}$ Tris buffer ( $\mathrm{pH} 8$ ) ranging from 5 to $200 \mu \mathrm{M}$, and $\mathrm{NaBH}_{4}$ (1 M stock in distilled water) was added to $1 \mathrm{mM}$ to reduce it to GS-menadiol, which was immediately used for enzyme assays. GS-hydroquinone (GSHQ) and GS-methylhydroquinone (GS-MHQ) were readily prepared by mixing GSH with $p$-benzoquinone (BQ) or methyl-p-benzoquinone (MBQ) in the $40 \mathrm{mM}$ Tris Buffer. The reactions were completed in less than $1 \mathrm{~min}$, and GS-MHQ and GS-HQ were stable for at least $30 \mathrm{~min}$ at room temperature and several hours on ice with no use of $\mathrm{NaBH}_{4}$.

UV/vis Spectrum Analysis. All reactions were done in 1 $\mathrm{mL}$ of $40 \mathrm{mM}$ Tris buffer ( $\mathrm{pH} 8$ ) with $1 \mathrm{mM} \mathrm{GSH}$ in quartz cuvette with $1 \mathrm{~cm}$ light path at $23{ }^{\circ} \mathrm{C}$. The spectra of $100 \mu \mathrm{M}$ GS-hydroquinones were recorded, and then $50 \mu \mathrm{g}$ of the tested GS-HQR was added. Spectra were continuously recorded at 1min intervals. The reactions were completed within $10 \mathrm{~min}$. The maximal changes after enzymatic reduction of GS-HHQ, GS-MHQ, GS-HQ and GS-menadiol were at 305, 307, 309, and $260 \mathrm{~nm}$, respectively. Thus, the enzymatic reactions were monitored by absorption changes at the wavelengths for the corresponding compounds. The absorption changes for the enzymatic conversion of $100 \mu \mathrm{M}$ GS-hydroquinones to hydroquinones were determined and used to calculate the reaction coefficients.

Kinetic Analysis. The enzymatic reactions were monitored at 305, 307, 309, and $260 \mathrm{~nm}$ for GS-HHQ GS-MHQ GS-HQ, and GS-menadiol, respectively, in $40 \mathrm{mM}$ Tris buffer $(\mathrm{pH} 8)$ at $23{ }^{\circ} \mathrm{C}$. The initial rates were calculated from the absorption 


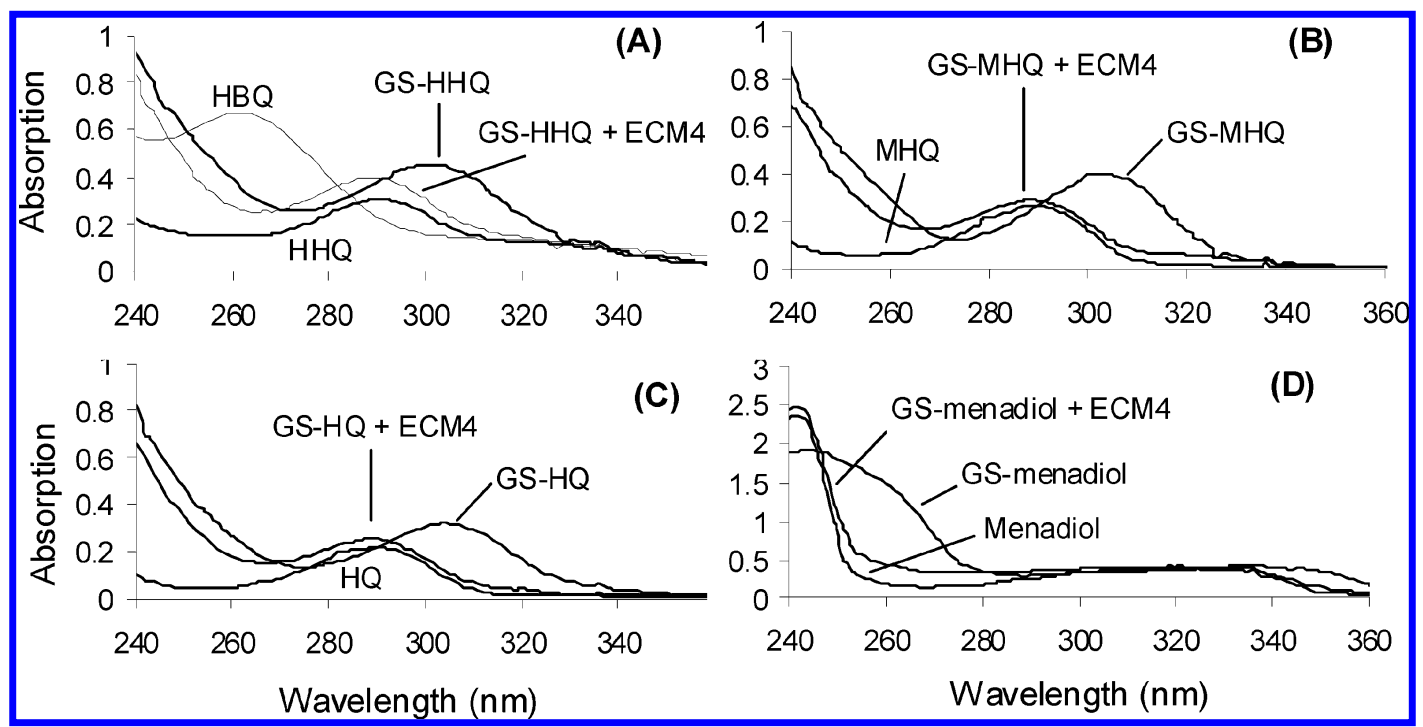

Figure 2. UV/vis spectra of enzymatic reduction of GS-hydroquinones. The reactions were performed in $1 \mathrm{~mL}$ of $40 \mathrm{mM}$ Tris buffer (pH 8) with hydroquinones and derivatives at $100 \mu \mathrm{M}$ and ECM4 at $50 \mu \mathrm{g} / \mathrm{mL}$ when added. (A) HHQ HBQ, GS-HHQ and addition of ECM4 released HHQ from GS-HHQ. (B) MHQ GS-MHQ and addition of ECM4 released MHQ from GS-MHQ. (C) HQ GS-HQ and addition of ECM4 released HQ from GS-HQ. (D) Menadiol, GS-menadiol, and addition of ECM4 released menadiol from GS-menadiol.

changes. GS-hydroquinones were tested in the range of less than its $K_{\mathrm{m}}$ value to four times of the $K_{\mathrm{m}}$ value, and GSH was fixed at $6 \mathrm{mM}$ for GS-HQ and GS-MHQ. When $\mathrm{NaBH}_{4}$ was added in reactions for GS-HHQ or GS-menadiol reduction, no additional GSH was added. The $K_{\mathrm{m}}$ values for $\mathrm{GSH}$ were determined for the tested enzymes with $600 \mu \mathrm{M}$ GS-MHQ and various concentrations of GSH. The rates and substrate concentrations were fitted to the Michaelis-Menten equation using nonlinear regression program GraFit 5 (Erithacus, Horley, Surrey) to obtain kinetic parameters.

High Performance Liquid Chromatography (HPLC) Analysis. Authentic hydroquinones, GS-hydroquinones, and enzyme-produced hydroquinones were further analyzed by HPLC (Waters, Milford, MA) equipped with a Nova-Pak C18 column $(3.9 \times 150 \mathrm{~mm}$, Waters $)$. The compounds were eluted by a gradient of acetonitrile in diluted trifluoroacetic acid $(0.1 \%)$ at $1 \mathrm{~mL} / \mathrm{min}$. The percentages of acetonitrile were as follows: 5 to $65 \%$, 5-mL linear gradient; $65 \%, 5 \mathrm{~mL}$; and $100 \%$, $4 \mathrm{~mL}$. The absorption spectra from 240 to $350 \mathrm{~nm}$ were monitored by a photodiode array detector operated with the Millennium 2010 program (Waters) on a personal computer. The compounds were identified by comparing their retention times and absorption spectra to those of authentic standards.

Nuclear Magnetic Resonance (NMR) Spectroscopy. ${ }^{1} \mathrm{H}$ NMR measurements were conducted on a Varian Inova 500 spectrometer with the operation frequency of $499.85 \mathrm{MHz}$. The ${ }^{1} \mathrm{H}$ NMR spectra of HHQ GS-HHQ and HHQ produced by PcpF from GS-HHQ were recorded at $10{ }^{\circ} \mathrm{C}$, averaging 112 scans for each spectrum. The spectra were referenced to sodium 2,2-dimethyl-2-silapentane-sulfonate, following the procedures reported elsewhere. ${ }^{23}$ All the NMR samples were prepared in $\mathrm{D}_{2} \mathrm{O}$. HHQ $(500 \mu \mathrm{M})$ was prepared in $40 \mathrm{mM}$ Tris, $\mathrm{pH} 8$, containing $1 \mathrm{mM} \mathrm{GSH}$ and $5 \mathrm{mM} \mathrm{NaBH}_{4}$. GSHHQ $(500 \mu \mathrm{M})$ was prepared by mixing $500 \mu \mathrm{M} H B Q$ which was obtained from HHQ autoxidation, and $1 \mathrm{mM} \mathrm{GSH}$ in 40 mM Tris $\mathrm{pH}$ 8. Immediately, $\mathrm{NaBH}_{4}$ was added to $5 \mathrm{mM}$. When $50 \mu \mathrm{g}$ of PcpF was added to $1 \mathrm{~mL}$ of GS-HHQ (500 $\mu \mathrm{M})$ solution, GS-HHQ was completely converted to HHQ in several minutes.

\section{RESULTS}

Nonenzymatic Formation and Enzymatic Reduction of GS-Hydroquinones. HBQ was not commercially available, so it was prepared from HHQ. HHQ had an absorption maximum at $290 \mathrm{~nm}$, and it was autoxidized to HBQ with an absorption maximum at $262 \mathrm{~nm}$. Upon addition of GSH, GSHHQ was immediately formed with an absorption maximum at $305 \mathrm{~nm}$. GS-HHQ was gradually autoxidized to a compound with absorption maximum at $350 \mathrm{~nm}$. Addition of $1 \mathrm{mM}$ $\mathrm{NaBH}_{4}$ prevented the autoxidation for at least $30 \mathrm{~min}$ at room temperature and several hours on ice. ECM4 (50 $\mu \mathrm{g}$ per $\mathrm{ml})$ reduced GS-HHQ back to HHQ within 1 min (Figure 2A). The spectrum of produced HHQ was similar to that of authentic HHQ (Figure 2A). Thus, the reaction started with $\mathrm{HHQ}$, which underwent autoxidation and then conjugation with GSH to form GS-HHQ and finally, ECM4 regenerated HHQ (Figure 1).

MBQ and BQ at $100 \mu \mathrm{M}$ had absorption peaks at 251 and $247 \mathrm{~nm}$, respectively (data not shown). When $1 \mathrm{mM}$ GSH was added, GS-MHQ and GS-HQ were immediately formed after mixing (Figure 2B,C). GS-MHQ had an absorption maximum at $307 \mathrm{~nm}$, and GS-HQ had the peak at $309 \mathrm{~nm}$. ECM4 (50 $\mu \mathrm{g} / \mathrm{mL}$ ) converted GS-MHQ and GS-HQ to MHQ and HQ at the expense of GSH. MHQ and HQ had similar absorption maxima at $290 \mathrm{~nm}$. The absorption spectra matched with those of MHQ and HQ obtained from MBQ and BQ reduction by 1 $\mathrm{mM} \mathrm{NaBH}_{4}$ (Figure 2B,C).

When $100 \mu \mathrm{M}$ menadione was mixed with $1 \mathrm{mM}$ GSH in the Tris buffer, reaction progressed very slowly. Consequently, higher concentrations of GSH and menadione were used. Directly mixing $20 \mu \mathrm{L}$ of $200 \mathrm{mM}$ GSH with $20 \mu \mathrm{L}$ of $25 \mathrm{mM}$ menadione allowed more than $95 \%$ completion after $30 \mathrm{~min}$ incubation. The produced GS-menadiol was autoxidized to GSmenadione during the conjugation, and the produced GSmenadione had an absorption maximum at $430 \mathrm{~nm}$, a characteristic peak of GS-menadione. ${ }^{24}$ The produced GSmenadione was added to the Tris buffer at a final concentration of $100 \mu \mathrm{M}$. After addition of $1 \mathrm{mM} \mathrm{GSH}$, there was no absorption change. Thus, $1 \mathrm{mM} \mathrm{GSH}$ did not result in any 


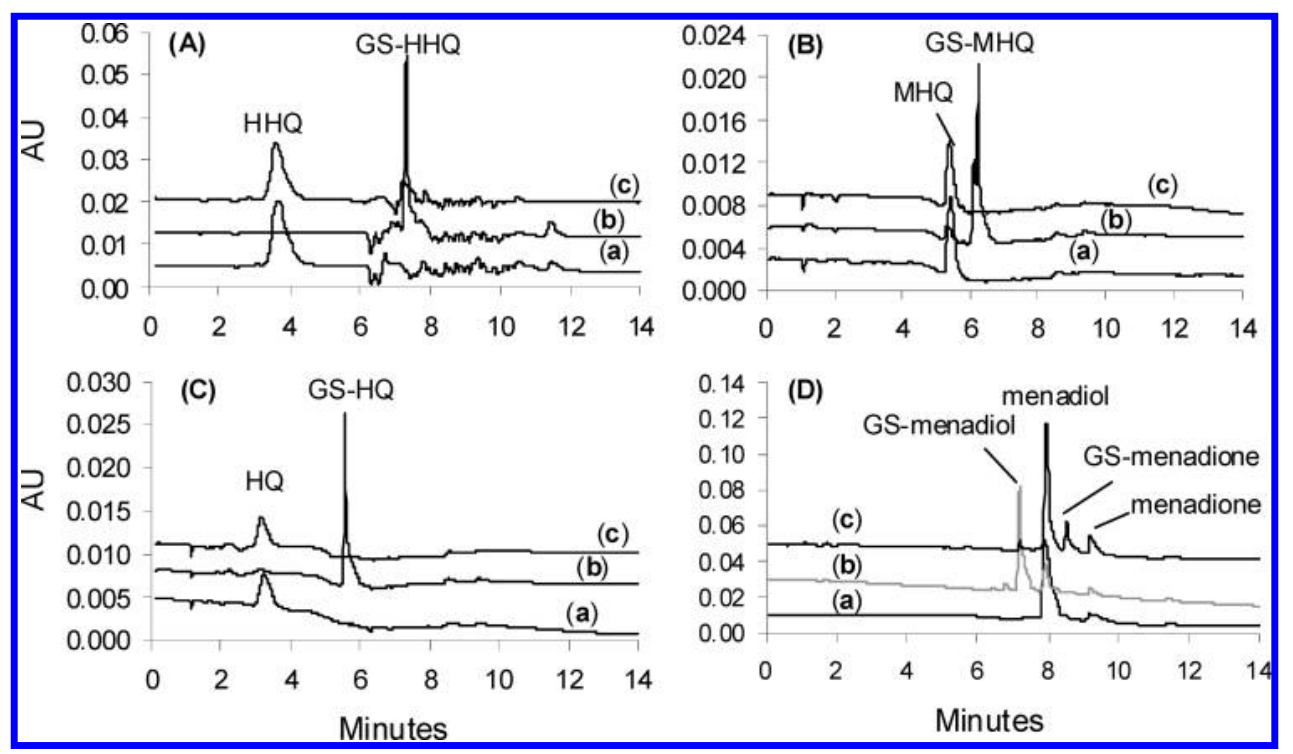

Figure 3. HPLC chromatograms of enzymatic reduction of GS-hydroquinones. The reaction conditions were the same as described in Figure 2 legend. (A) HHQ (a), GS-HHQ (b), and HHQ released from GS-HHQ by ECM4 (c). (B) MHQ (a), GS-MHQ (b), and MHQ released from GSMHQ by ECM4 (c). (C) HQ (a), GS-HQ (b), and HQ released from GS-HQ by ECM4 (c). (D) Menadiol (a), GS-menadiol (b), and menadiol released from GS-menadiol by ECM4 (c). The chromatograms $(a, b, c)$ are separated by moving the $y$-axis for (b) and (c).

apparent reduction of GS-menadione to GS-menadiol. When $50 \mu \mathrm{g}$ of ECM4 was added, no apparent change of absorption at $430 \mathrm{~nm}$ was observed, indicating the enzyme did not use GSmenadione as a substrate (data not shown). In a separate experiment, $100 \mu \mathrm{M}$ GS-menadione was first reduced by addition of $1 \mathrm{mM} \mathrm{NaBH}_{4}$ to GS-menadiol that had an absorption peak at $240 \mathrm{~nm}$ and a shoulder at $260 \mathrm{~nm}$ (Figure 2D). Then, ECM4 $(50 \mu \mathrm{g})$ converted GS-menadiol to menadiol (Figure 2D) within $1 \mathrm{~min}$. Interestingly, adding GSH to $1 \mathrm{mM}$ did not stimulate the reaction rate. Since the reaction contained about $300 \mu \mathrm{M}$ GSH originated from the GSmenadione preparation, we could not conclude whether GSH was required for the reaction. GS-menadione was subsequently prepared with equal molar ratio of menadione and GSH. Then GS-menadione was reduced to GS-menadiol by $\mathrm{NaBH}_{4}$. Again ECM4 converted GS-menadiol to menadiol. When GS-HQ was prepared from equal molar of GSH and BQ, ECM4 did not use it unless additional GSH or $\mathrm{NaBH}_{4}$ was added. Thus, GSHQRs also used $\mathrm{NaBH}_{4}$ to convert GS-hydroquinones to hydroquinones.

On the basis of spectral analysis, PcpF, EcYqjG, and CnYqjG also used GS-HHQ, GS-HQ, GS-MHQ and GS-menadiol as substrates (data not shown). hGSTO1-1 did not use any of the GS-hydroquinones. Other thiols were also used to react with benzoquinones, forming L-cysteinyl-hydroquinones, 2-mercaptoethanol-S-hydroquinones, and dithiothreitol-S-hydroquinones. The tested GS-HQRs did not use these conjugates as substrates in the presence or absence of $1 \mathrm{mM} \mathrm{GSH}$.

HPLC Analysis of the Spontaneous Formation and Enzymatic Breakdown of GS-Hydroquinones. In conjunction with the spectral analysis (Figure 2), HPLC was also used to confirm the formation and the enzymatic reduction of the GS-hydroquinones. Authentic HHQ gave a retention time of $3.7 \mathrm{~min}$ with an absorption maximum at $288 \mathrm{~nm}$ (Figure 3A, chromatogram a). GS-HHQ had a retention time of $7.6 \mathrm{~min}$ with an absorption maximum at $301 \mathrm{~nm}$ (Figure 3A, chromatogram $b$ ). The hypsochromic shift in the absorption maxima from the UV/vis spectrum (Figure 2a) was possibly due to the low $\mathrm{pH}$ in the HPLC running buffer containing trifluoroacetic acid. After treatment with ECM4, the peak of GS-HHQ completely disappeared, and the peak of HHQ came back (Figure 3A, chromatogram c). Nonenzymatic reduction of GS-HHQ was not detectable.

MHQ produced from MBQ reduction by $\mathrm{NaBH}_{4}$, had a retention time of $5.4 \mathrm{~min}$ with an absorption maximum at 288 nm (Figure 3B, chromatogram a). GS-MHQ had a retention time of $6.2 \mathrm{~min}$ with an absorption maximum at $302 \mathrm{~nm}$ (Figure 3B, chromatogram b). After treatment with ECM4, the peak of GS-MHQ completely disappeared, and the peak of MHQ appeared (Figure 3B, chromatogram c). Nonenzymatic reduction was not detectable.

$\mathrm{HQ}$ produced from BQ reduction by $\mathrm{NaBH}_{4}$, had a retention time of $3.2 \mathrm{~min}$ with an absorption maximum at $288 \mathrm{~nm}$ (Figure 3C, chromatogram a). GS-HQ had a retention time of $5.5 \mathrm{~min}$ with an absorption maximum at $304 \mathrm{~nm}$ (Figure 3C, chromatogram b). After treatment with ECM4, the peak of GS-HQ was completely gone, and the peak of HQ showed up (Figure 3C, chromatogram c). Nonenzymatic reduction was not detectable.

Menadiol, produced from menadione by $\mathrm{NaBH}_{4}$ reduction, had a retention time of 7.9 min with a sharp peak at $243 \mathrm{~nm}$ (Figure 3D, chromatogram a). In the chromatogram, a small amount of menadione was eluted off the column at $9.2 \mathrm{~min}$ with a peak at $248 \mathrm{~nm}$ and a shoulder at $262 \mathrm{~nm}$. GS-menadiol had a retention time of $7.2 \mathrm{~min}$ with an absorption shoulder at $260 \mathrm{~nm}$ (Figure 3D, chromatogram b). After treatment with ECM4, the peak of GS-menadiol disappeared, and the peak size of menadiol significantly increased (Figure 3D, chromatogram c). Interestingly, there was a small peak of GS-menadione at 8.6 min with absorption maximum at $251 \mathrm{~nm}$ in the ECM4-treated sample. We believe that GS-menadione was formed from autoxidation of GS-menadiol during incubation, and ECM4 did not use it.

NMR Spectra of $\mathrm{HHQ}$ and GS-HHQ. The ${ }^{1} \mathrm{H}$ NMR spectrum of HHQ (Figure 4A) showed three major peaks at the chemical shifts of $6.30 \mathrm{ppm}$ (doublet of doublet (dd), $J_{56}=$ 


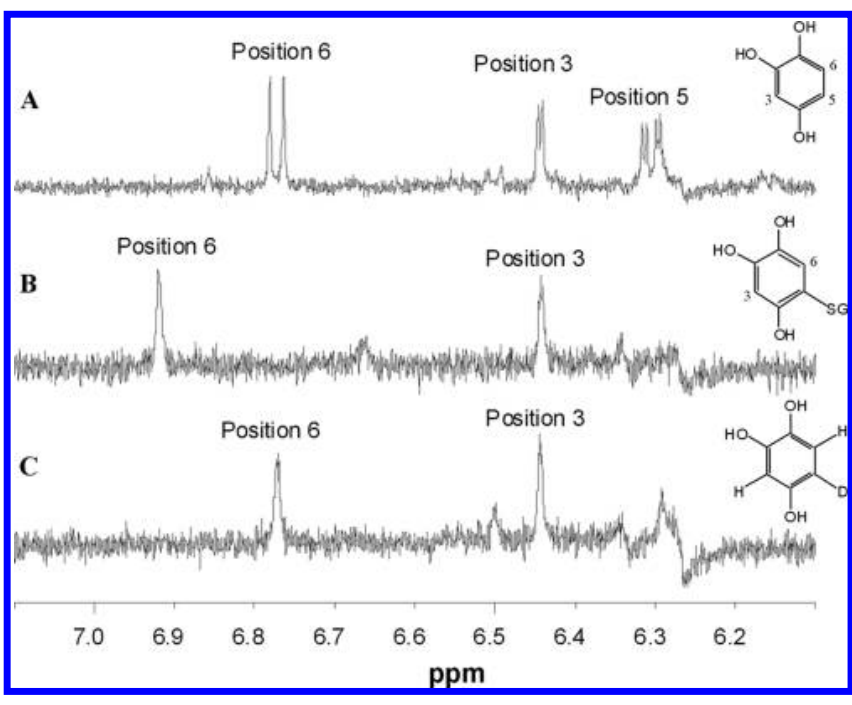

Figure 4. ${ }^{1} \mathrm{H}$ NMR spectra of HHQ and GS-HHQ. Only the aromatic region of the ${ }^{1} \mathrm{H}$ NMR spectra was shown. (A) $500 \mu \mathrm{M}$ of $\mathrm{HHQ}$ showing three major peaks. (B) GS-HHQ stabilized with $\mathrm{NaBH}_{4}$, having two major peaks. (C) HHQ released from GS-HHQ by PcpF in $\mathrm{D}_{2} \mathrm{O}$, revealing two aromatic protons with deuterium undetectable.

$\left.8.5 \mathrm{~Hz}, J_{53}=2.7 \mathrm{~Hz}\right), 6.44 \mathrm{ppm}\left(\right.$ doublet $\left.(\mathrm{d}), J_{35}=2.7 \mathrm{~Hz}\right)$, and $6.77 \mathrm{ppm}$ (doublet $(\mathrm{d}), J_{65}=8.5 \mathrm{~Hz}$ ), corresponding to the NMR of three aromatic protons in HHQ. The obtained spectrum was in agreement with the Aldrich published ${ }^{1} \mathrm{H}$ spectrum of $\mathrm{HHQ}^{25}$ and more importantly, the multiplicities of individual protons were quite well explained by their positions on the aromatic ring with mutually agreed $J$ coupling constants. The doublet-of-doublet at $6.30 \mathrm{ppm}$ represented the NMR signal of the proton at position 5 of $\mathrm{HHQ}$ forming from the spin-spin interactions of this proton with other two protons at positions $3\left(J_{53}=2.7 \mathrm{~Hz}\right)$ and $6\left(J_{56}=8.5 \mathrm{~Hz}\right)$, respectively. The doublet at $6.44 \mathrm{ppm}$ referred to the proton at position 3 . The doublet splitting was attributed to the spin-spin coupling of this proton to the proton at position 5. Because these two interacting protons were structurally distant, their spin-spin coupling was expected to be relatively weak, resulting in a small $J$ constant, $J_{35}=2.7$. The doublet at $6.77 \mathrm{ppm}$ referred to the proton at position 6 with $J_{65}=8.5 \mathrm{~Hz}$. This relatively large coupling constant was reasoned by the strong spin-spin interaction of this proton and its adjacent proton at position 5 . Typically, such a coupling generates a $J$ constant of $4-10 \mathrm{~Hz}^{26}$

The ${ }^{1} \mathrm{H}$ spectrum of GS-HHQ was shown in Figure 4B. Comparison of this spectrum to the HHQ spectrum (Figure 4A) revealed that one ${ }^{1} \mathrm{H}$ peak of $\mathrm{HHQ}$ was lost and no multiplicities existed for the remaining two ${ }^{1} \mathrm{H}$ peaks. The spectrum matched the effect of GS-conjugation, in which a glutathionyl group replaced the proton at position 5 . Accordingly, the NMR peak of the position 5 proton $(6.30$ ppm) disappeared, and the remaining two protons at parapositions to each other showed no apparent spin-spin coupling. Importantly, the peak displacements of remaining protons further confirmed the above assumption. As assumed, the replacement occurred at position 5, closer to the position 6 than to the position 3. As a result, the substituting glutathionyl group should have a large effect on the magnetic environment of the position 6 proton but little on that of the position 3 proton. This was demonstrated by the fact that the peak of the position 6 proton was largely displaced to the downfield $(\Delta \delta=$
$0.15 \mathrm{ppm})$, but the peak of the position 3 proton was not displaced (Figure 4A,B).

Figure 4C depicts the ${ }^{1} \mathrm{H}$ spectrum of $\mathrm{HHQ}$ produced by PcpF from GS-HHQ. As mentioned in the Experimental Procedures, because the sample was prepared in $\mathrm{D}_{2} \mathrm{O}$, the hydrogen at position 5 of the produced HHQ should be a deuterium after the removal of the glutathionyl group by PcpF. Therefore, no signal of the position 5 nucleus can be measured for the ${ }^{1} \mathrm{H}$ NMR. The obtained ${ }^{1} \mathrm{H}$ spectrum (Figure $4 \mathrm{C}$ ) clearly demonstrated the above hypothesis. Two single ${ }^{1} \mathrm{H}$ peaks of the product (Figure 4C) were observed at the same chemical shifts as those of the starting HHQ (Figure 4A). We believe that they represented the NMR of protons at positions 6 and 3 of the produced HHQ. Again, these two protons were at the para-positions to each other, and no spin-spin splitting was observed.

Enzymatic Assay Development. As shown in Figure 2, GS-HHQ GS-MHQ GS-HQ and GS-menadiol had absorption maxima at 305, 307, 309, and $260 \mathrm{~nm}$, respectively. GSH did not spontaneously reduce GS-hydroquinones, and the reaction started upon adding ECM4 (Figure 5). After

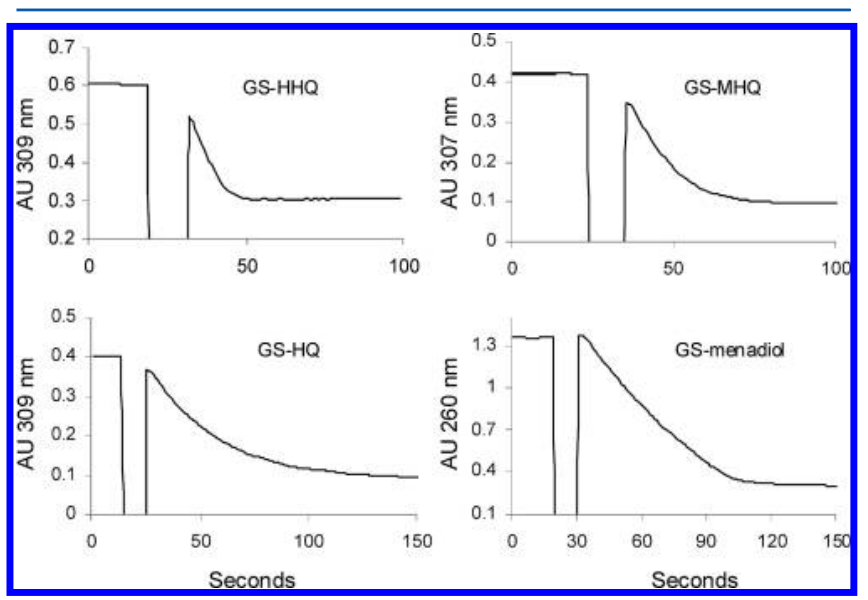

Figure 5. Monitoring the reduction of GS-hydroquinones by GSHQRs. The reactions were done in Tris buffer with the tested substrate at a target concentration of $100 \mu \mathrm{M}$ in $1 \mathrm{~mL}$ of reaction volume. The absorption was monitored without enzyme for 10-30 s, and then ECM4 was added at 50, 25, 25, and $12 \mu \mathrm{g}$ for GS-HHQ GSMHQ GS-HQ and GS-menadiol, respectively, to start the reaction.

enzymatic reduction, the maximal decreases in absorption were at the absorption maxima for the corresponding GShydroquinones. The enzymatic activities were monitored for the changes at the wavelengths of absorption maxima. The rates of absorption changes gradually slowed to zero when the reaction approached completion. Thus, the enzymatic reduction of GS-HHQ GS-MHQ GS-HQ and GS-menadiol were monitored at 305, 307, 309, and $260 \mathrm{~nm}$, respectively. The reaction extinction coefficients were determined from the absorption changes for $100 \mu \mathrm{M}$ GS-hydroquinones before and after complete enzymatic reduction at the corresponding absorption maxima. The reaction coefficients were $\varepsilon_{309}$ (GSHQ) for $2.64 \pm 0.14 \mathrm{~cm}^{-1} \mathrm{mM}^{-1}, \varepsilon_{307}$ (GS-MHQ) for $2.97 \pm$ $0.09 \mathrm{~cm}^{-1} \mathrm{mM}^{-1}, \varepsilon_{305}$ (GS-HHQ) for $2.15 \pm 0.02 \mathrm{~cm}^{-1} \mathrm{mM}^{-1}$, and $\varepsilon_{260}$ (GS-menadiol) for $10.3 \pm 0.45 \mathrm{~cm}^{-1} \mathrm{mM}^{-1}$. The values were averages of at least three samples with standard deviations.

Kinetic Analysis. The apparent kinetic parameters of four GS-HQRs were tested using GS-HHQ, GS-MHQ GS-HQ and 
Table 1. Apparent Kinetic Parameters of GS-HQRs

\begin{tabular}{|c|c|c|c|c|}
\hline kinetics ${ }^{a} /$ enzyme & CnYqjG & EcYqjG & ECM4 & PcpF \\
\hline \multicolumn{5}{|c|}{ Various GSH with $600 \mu \mathrm{M}$ GS-MHQ } \\
\hline$k_{\text {cat }}\left(\mathrm{s}^{-1}\right)$ & $37 \pm 1.5$ & $15 \pm 0.5$ & $7.5 \pm 0.3$ & $14 \pm 0.3$ \\
\hline$K_{\mathrm{m} \cdot \mathrm{GSH}}(\mu \mathrm{M})$ & $880 \pm 110$ & $840 \pm 100$ & $350 \pm 73$ & $1200 \pm 72$ \\
\hline$k_{\mathrm{cat}} / K_{\mathrm{m}}\left(\mathrm{M}^{-1} \mathrm{~s}^{-1}\right)$ & $42 \times 10^{3}$ & $18 \times 10^{3}$ & $21 \times 10^{3}$ & $12 \times 10^{3}$ \\
\hline \multicolumn{5}{|c|}{ Various GS-MHQ with $6 \mathrm{mM}$ GSH } \\
\hline$k_{\text {cat }}\left(\mathrm{s}^{-1}\right)$ & $31 \pm 1.7$ & $15 \pm 0.6$ & $8.4 \pm 0.7$ & $14 \pm 1.1$ \\
\hline$K_{\mathrm{m} . \mathrm{GS}-\mathrm{MHQ}}(\mu \mathrm{M})$ & $190 \pm 26$ & $70 \pm 10$ & $150 \pm 44$ & $34 \pm 11$ \\
\hline$k_{\mathrm{cat}} / K_{\mathrm{m}}\left(\mathrm{M}^{-1} \mathrm{~s}^{-1}\right)$ & $160 \times 10^{3}$ & $210 \times 10^{3}$ & $56 \times 10^{3}$ & $410 \times 10^{3}$ \\
\hline \multicolumn{5}{|c|}{ Various GS-HQ with $6 \mathrm{mM}$ GSH } \\
\hline$k_{\text {cat }}\left(\mathrm{s}^{-1}\right)$ & $22 \pm 1.9$ & $14 \pm 2.3$ & $13 \pm 1.6$ & $11 \pm 1.5$ \\
\hline$K_{\mathrm{m} . \mathrm{GS}-\mathrm{HQ}}(\mu \mathrm{M})$ & $240 \pm 70$ & $390 \pm 130$ & $370 \pm 120$ & $430 \pm 140$ \\
\hline$k_{\mathrm{cat}} / K_{\mathrm{m}}\left(\mathrm{M}^{-1} \mathrm{~s}^{-1}\right)$ & $91 \times 10^{3}$ & $36 \times 10^{3}$ & $35 \times 10^{3}$ & $26 \times 10^{3}$ \\
\hline \multicolumn{5}{|c|}{ Various GS-HHQ with $1 \mathrm{mM} \mathrm{NaBH}_{4}$} \\
\hline$k_{\text {cat }}\left(\mathrm{s}^{-1}\right)$ & $4.4 \pm 0.5$ & $7.2 \pm 0.8$ & $7.1 \pm 0.5$ & $19 \pm 1.5$ \\
\hline$K_{\mathrm{m} . \mathrm{GS}-\mathrm{HHQ}}(\mu \mathrm{M})$ & $34 \pm 9$ & $35 \pm 10$ & $12 \pm 3$ & $130 \pm 18$ \\
\hline$k_{\mathrm{cat}} / K_{\mathrm{m}}\left(\mathrm{M}^{-1} \mathrm{~s}^{-1}\right)$ & $130 \times 10^{3}$ & $210 \times 10^{3}$ & $590 \times 10^{3}$ & $15 \times 10^{3}$ \\
\hline \multicolumn{5}{|c|}{ Various GS-menadiol with $1 \mathrm{mM} \mathrm{NaBH} 4$} \\
\hline$k_{\text {cat }}\left(\mathrm{s}^{-1}\right)$ & $13 \pm 1.5$ & $4.9 \pm 0.6$ & $14 \pm 2.2$ & $4.6 \pm 0.1$ \\
\hline$K_{\text {m.GS-menadiol }}(\mu \mathrm{M})$ & $17 \pm 4.5$ & $4.0 \pm 1.6$ & $6.7 \pm 3.2$ & $3.4 \pm 0.1$ \\
\hline$k_{\mathrm{cat}} / K_{\mathrm{m}}\left(\mathrm{M}^{-1} \mathrm{~s}^{-1}\right)$ & $760 \times 10^{3}$ & $1200 \times 10^{3}$ & $2100 \times 10^{3}$ & $1400 \times 10^{3}$ \\
\hline
\end{tabular}

${ }^{a_{T}}$ The $K_{\mathrm{m}}$ values for GS-hydroquinones were determined for each enzyme with fixed GSH at $6 \mathrm{mM}$ or $\mathrm{NaBH}_{4}$ at $1 \mathrm{mM}$, and the $K_{\mathrm{m}}$ values for GSH were determined with fixed GS-MHQ at $600 \mu \mathrm{M}$.

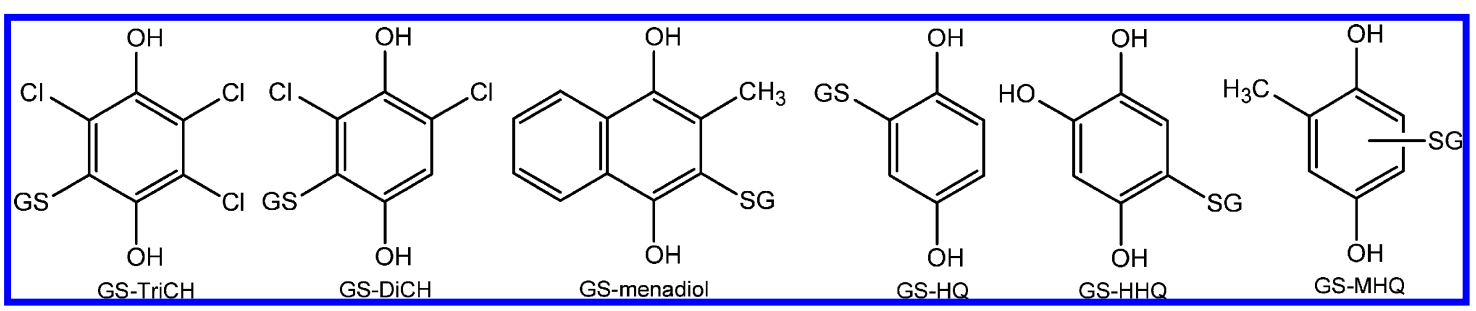

Figure 6. Structures of GS-HQRs' substrates. GS-TriCH, GS-trichloro-p-hydroquinone; GS-DiCH, GS-dichloro- $p$-hydroquinone; GS-HQ, GS-phydroquinone; GS-HHQ GS-hydroxy-p-hydroquinone; GS-MHQ GS-methyl-p-hydroquinone.

GS-menadiol (Table 1). GS-MHQ and GS-HQ were stable during the assay, and $\mathrm{NaBH}_{4}$ was not added to the reaction mixture. GSH was required for the enzymes to reduce GSMHQ and GS-HQ. When GS-MHQ was the fixed substrate at $600 \mu \mathrm{M}$, the $K_{\mathrm{m}}$ values for GSH were $0.88,0.84,0.35$, and 1.15 $\mathrm{mM}$ for CnYqjG, EcYqjG, ECM4, and PcpF, respectively (Table 1). When GSH was the fixed substrate at $6 \mathrm{mM}$, the apparent kinetic parameters for GS-MHQ and GS-HQ were determined. The $K_{\mathrm{m}}$ values ranged from 34 to $190 \mu \mathrm{M}$ of GSMHQ and from 237 to $427 \mu \mathrm{M}$ of GS-HQ for the tested enzymes. When using fixed GS-HHQ or GS-menadiol to determine the $K_{\mathrm{m}}$ values for GSH, GSH was not required due to the presence of $\mathrm{NaBH}_{4}$. The $1 \mathrm{mM} \mathrm{NaBH}_{4}$ added to prevent autoxidation of GS-HHQ and GS-menadiol was able to provide the reducing power for the enzymatic reduction. Without the enzymes, $\mathrm{NaBH}_{4}$ could not reduce GS-hydroquinones. Thus, the apparent kinetic parameters for GS-HHQ and GS-menadiol were determined without additional GSH.

\section{DISCUSSION}

GS-HQRs reduced several GS-hydroquinones at the expense of GSH or $\mathrm{NaBH}_{4}$. The enzymatic reactions were confirmed with $\mathrm{UV} /$ vis spectrometry (Figure 2) and HPLC analysis (Figure 3) on the basis of retention times and UV/vis spectra in comparison with authentic standards. Further, the reduction of GS-HHQ to HHQ by PcpF was confirmed by NMR analysis; replacement of the glutathionyl group with deuterium in $\mathrm{D}_{2} \mathrm{O}$ unequivocally confirmed the reaction (Figure 4). The NMR results also showed that 5-GS-2-hydroxy-p-hydroquinone was the only detectable product from the spontaneous reaction between HBQ and GSH.

Previously, the $P$. chrysosporium GS-HQR (PcGHR1) was shown to reduce GS-menadione to menadione by HPLC analysis. ${ }^{3}$ PcGHR1 has a high $K_{\mathrm{m}}$ value of $2 \mathrm{mM}$ toward GSmenadione, suggesting that high GS-menadione concentrations were used in reactions. In contrast, we found that GSmenadione was not a substrate of the tested GS-HQRs, but its reduced form, GS-menadiol, was the substrate. The tested GSHQRs all had low $K_{\mathrm{m}}$ values of 3-17 $\mu \mathrm{M}$ for GS-menadiol (Table 1). The discrepancy may be real or PcGHR1 may have reduced trace GS-menadiol present in the reaction mixture to menadiol, which is autoxidized to menadione before or during HPLC analysis, as we showed that menadiol and GS-menadiol were readily autoxidized to menadione and GS-menadione via HPLC analysis (Figure 3D). Our evidence indicates that the enzymes are able to use GS-hydroquinones but not GSbenzoquinones.

GS-HQRs are believed to release hydroquinones and transfer the glutathionyl group to a Cys residue at the catalytic site, forming protein-glutathione mixed disulfide bond. A GSH comes in to regenerate the protein and release GS-SG to complete the reaction. ${ }^{1,3}$ Small thiols, such as dithiothreitol, L- 
cysteine, and 2-mercaptoethanol, can replace GSH to regenerate the enzymes. ${ }^{1}$ This is similar to the proposed mechanism by which hGSTO1-1 reduces $S$-(phenacyl) glutathiones. ${ }^{5}$ Here, we found that $\mathrm{NaBH}_{4}$ also regenerated the enzymes. This is not a surprise since $\mathrm{NaBH}_{4}$ is known to reduce protein-glutathione mixed disulfide bond. ${ }^{27}$ However, $\mathrm{NaBH}_{4}$ did not directly reduce the sulfur-carbon bond in GShydroquinones.

Because the tested GS-HQRs all use GS-TriCH as a substrate, ${ }^{1}$ we have suggested that they can use other GShydroquinones. Here, we demonstrated that the four tested GS-HQRs used several GS-hydroquinones with different substitutions (Figure 6). The aromatic ring is completely substituted for GS-TriCH and GS-menadiol, whereas GS-HQ has no additional substitution. The GS-hydroquinones have different substitutions, including chlorine, methyl group (GSMHQ), hydroxyl group (GS-HHQ), and aromatic ring (GSmenadiol). Given the diverse origins of the four enzymes from E. coli, C. necator, S. chlorophenolicum, and S. cerevisiae with sequence identity as low as $35.4 \%,{ }^{1}$ we predict that most GSHQRs should behave similarly as the four tested GS-HQRs and be able to use a variety of GS-hydroquinones as substrates. The prediction is in agreement with the report that a GS-HQR from P. chrysosporium reduces GS-HQ to HQ. ${ }^{3}$ Because all the tested GS-HQRs are able to reduce GS-hydroquinones, we rename the enzymes as $S$-glutathionyl-p-hydroquinone reductases (GSHQRs), which is also used to describe the enzyme (PcGHR1) of $P$. chrysosporium. ${ }^{3}$ Collectively, our data support the proposal of a new GST class xi for GS-HQRs. ${ }^{3}$

Kinetic data revealed the activity difference among the enzymes toward the tested substrates (Table 1). If we compare the $k_{\text {cat }} / K_{\mathrm{m}}$ values for the reactions with the same reducing power as GSH or $\mathrm{NaBH}_{4}$, it becomes clear that the enzymes prefer hydrophobic, bulky GS-menadiol to hydrophilic, smaller GS-HHQ or prefer bulky GS-MHQ to smaller GS-HQ. However, the specific enzyme activity varied among tested enzymes.

The physiological role of PcpF in the maintenance of PCP degradation pathway in S. chlorophenolicum is well established; ${ }^{6}$ however, the physiological roles of GS-HQRs have not been demonstrated in other organisms despite their common presence in bacteria, fungi, and plants. Given the tendency of hydroquinone autoxidation and the spontaneous reaction of benzoquinones with GSH to form GS-hydroquinones, it is not hard to imagine the formation of GS-hydroquinones from hydroquinones and benzoquinones that are metabolic intermediates. Formation of GS-hydroquinones reduces the efficiency of the metabolic pathway, ${ }^{6}$ and GS-hydroquinones are more easily autoxidized than the corresponding hydroquinones, ${ }^{15}$ leading to elevated ROS that are detrimental to cells. PcpF and other GS-HQRs convert GS-hydroquinones to hydroquinones, which can reenter the metabolic pathway, minimizing toxic effects to the cells and maximizing the efficiency of the metabolism. Further, GS-HQRs may participate in quinone reduction (Figure 1). Benzoquinones can be reduced by quinone reductases that consume one $\mathrm{NAD}(\mathrm{P}) \mathrm{H}$ (Figure 1, step 1 reverse reaction). Benzoquinones can also react with GSH to form GS-hydroquinones that can be further reduced by GS-HQRs to regenerate hydroquinones with the production of GS-SG (Figure 1, steps 2 and 3). Glutathione reductase reduces the latter back to two GSH molecules with the consumption of one $\mathrm{NAD}(\mathrm{P}) \mathrm{H}$. Both reactions consume a total of one $\mathrm{NAD}(\mathrm{P}) \mathrm{H}$. $\mathrm{HBQ}$ is a metabolic intermediate of 2,4,5-T biodegradation in Burkholderia cepacia AC $1100 .^{28}$ It is possible that both a quinone reductase and a GS-HQR participate in its reduction to HHQ (Figure 1). Thus, GS-HQRs may play two functional rolesmetabolic pathway maintenance and quinone reduction in pathways with hydroquinones or benzoquinones as intermediates.

\section{AUTHOR INFORMATION}

\section{Corresponding Author}

*Telephone: (509) 335-2787. Fax: (509) 335-4159. E-mail: xun@mail.wsu.edu.

\section{Funding}

The work was supported by the U.S. National Science Foundation Grant MCB-1021148. L.K.M.L. was partially supported by the WSU College of Science Undergraduate Student Research Minigrant. P.G.B. was funded by a grant from the Australian National Health and Medical Research Council.

\section{Notes}

The authors declare no competing financial interest.

\section{ABBREVIATIONS USED}

GS-HQRs, S-glutathionyl-hydroquinone reductases; GSH, glutathione; GS, glutathionyl; TriCH, trichloro- $p$-hydroquinone; $\mathrm{DiCH}$, dichloro- $p$-hydroquinone; GS-HHQ GS-hydroxy-p-hydroquinone; GS-MHQ GS-methyl-p-hydroquinone; GS-HQ GS-p-hydroquinone

\section{REFERENCES}

(1) Xun, L., Belchik, S., Xun, R., Huang, Y., Zhou, H., Sanchez, E., Kang, C., and Board, P. P. (2010) S-Glutathionyl-(chloro)hydroquinone reductases: a novel class of glutathione transferases. Biochem. J. 428, 419-427.

(2) Dixon, D. P., Davis, B. G., and Edwards, R. (2002) Functional divergence in the glutathione transferase superfamily in plants. Identification of two classes with putative functions in redox homeostasis in Arabidopsis thaliana. J. Biol. Chem. 277, 30859-30869.

(3) Meux, E., Prosper, P., Ngadin, A., Didierjean, C., Morel, M., Dumarçay, S., Lamant, T., Jacquot, J. P., Favier, F., and Gelhaye, E. (2011) Glutathione transferases of Phanerochaete chrysosporium: Sglutathionyl-p-hydroquinone reductase belongs to a new structural class. J. Biol. Chem. 286, 9162-9173.

(4) Whitbread, A. K., Masoumi, A., Tetlow, N., Schmuck, E., Coggan, M., and Board, P. G. (2005) Characterization of the omega class of glutathione transferases. Methods Enzymol. 401, 78-99.

(5) Board, P. G., and Anders, M. W. (2007) Glutathione transferase omega 1 catalyzes the reduction of $S$-(phenacyl)glutathiones to acetophenones. Chem. Res. Toxicol. 20, 149-154.

(6) Huang, Y., Xun, R., Chen, G., and Xun, L. (2008) Maintenance role of a glutathionyl-hydroquinone lyase $(\mathrm{PcpF})$ in pentachlorophenol degradation by Sphingobium chlorophenolicum ATCC 39723. J. Bacteriol. 190, 7595-7600.

(7) Orser, C. S., Dutton, J., Lange, C., Jablonski, P., Xun, L., and Hargis, M. (1993) Characterization of a Flavobacterium glutathione $S$ transferase gene involved in reductive dechlorination. J. Bacteriol. 175, 2640-2644.

(8) Xun, L., Topp, E., and Orser, C. S. (1992) Purification and characterization of a tetrachloro- $p$-hydroquinone reductive dehalogenase from a Flavobacterium sp. J. Bacteriol. 174, 8003-8007.

(9) McCarthy, D. L., Navarrete, S., Willett, W. S., Babbitt, P. C., and Copley, S. D. (1996) Exploration of the relationship between tetrachlorohydroquinone dehalogenase and the glutathione $S$-transferase superfamily. Biochem. 35, 14634-14642.

(10) Crosby, D. G. (1981) Environmental chemistry of pentachlorophenol. Pure Appl. Chem. 53, 1052-1080. 
(11) Belchik, S. M., and Xun, L. (2011) S-Glutathionyl-(chloro)hydroquinone reductases: a new class of glutathione transferases functioning as oxidoreductases. Drug Metab. Rev. 43, 307-316.

(12) Hill, B. A., Kleiner, H. E., Ryan, E. A., Dulik, D. M., Monks, T. J., and Lau, S. S. (1993) Identification of multi-S-substituted conjugates of hydroquinone by HPLC-coulometric electrode array analysis and mass spectroscopy. Chem. Res. Toxicol. 6, 459-469.

(13) Buffinton, G. D., Ollinger, K., Brunmark, A., and Cadenas, E. (1989) DT-diaphorase-catalysed reduction of 1,4-naphthoquinone derivatives and glutathionyl-quinone conjugates. Effect of substituents on autoxidation rates. Biochem. J. 257, 561-571.

(14) Kumagai, Y., Koide, S., Taguchi, K., Endo, A., Nakai, Y., Yoshikawa, T., and Shimojo, N. (2002) Oxidation of proximal protein sulfhydryls by phenanthraquinone, a component of diesel exhaust particles. Chem. Res. Toxicol. 15, 483-489.

(15) Brunmark, A., and Cadenas, E. (1988) Reductive addition of glutathione to $p$-benzoquinone, 2-hydroxy-p-benzoquinone, and $p$ benzoquinone epoxides. Effect of the hydroxy- and glutathionylsubstituents on $p$-benzohydroquinone autoxidation. Chem. Biol. Interact. 68, 273-298.

(16) Monks, T. J., Lau, S. S., Highet, R. J., and Gillette, J. R. (1985) Glutathione conjugates of 2-bromohydroquinone are nephrotoxic. Drug Metab. Dispos. 13, 553-559.

(17) Mertens, J. J., Gibson, N. W., Lau, S. S., and Monks, T. J. (1995) Reactive oxygen species and DNA damage in 2-bromo-(glutathion-Syl) hydroquinone-mediated cytotoxicity. Arch. Biochem. Biophys. 320, $51-58$.

(18) Kari, F. W., Bucher, J., Eustis, S. L., Haseman, J. K., and Huff, J. E. (1992) Toxicity and carcinogenicity of hydroquinone in F344/N rats and B6C3F1 mice. Food Chem. Toxicol. 30, 737-747.

(19) Ishiguro, S., Saugawara, H., Kusama, M., Yano, S., Shimojima, N., and Sugawara, S. (1976) Glass capillary column gas chromatographic analysis of tobacco and cellulose cigarette smoke. I. Acidic fractions. Sci. Pap. Cent. Res. Inst. Jpn. Tob. Salt Public Corp. 118, 207211.

(20) Wefers, H., and Sies, H. (1983) Hepatic low-level chemiluminescence during redox cycling of menadione and the menadione-glutathione conjugate: relation to glutathione and NAD(P)H:quinone reductase (DT-diaphorase) activity. Arch. Biochem. Biophys. 224, 568-578.

(21) Belchik, S. M., and Xun, L. (2011) S-Glutathionyl-(chloro)hydroquinone reductases: a new class of glutathione transferases functioning as oxidoreductases. Drug Metab. Rev. 43, 307-316.

(22) Bohuslavek, J., Chanama, S., Crawford, R. L., and Xun, L. (2005) Identification and characterization of hydroxyquinone hydratase activities from Sphingobium chlorophenolicum ATCC 39723. Biodegradation 16, 353-362.

(23) Wishart, D. S., Bogam, C. G., Yao, J., Abildgard, F., Dyson, H. J., Oldfield, E., Markley, J. L., and Sykes, B. D. (1995) 1H, 13C and 15N chemical shift referencing in biomolecular NMR. J. Biomol. NMR 6, 135-140.

(24) Nickerson, W. J., Falcone, G., and Strauss, G. (1963) Studies on quinone-thioethers. I. Mechanism of formation and properties of thiodione. Biochemistry 2, 537-543.

(25) Pouchert, C. (1992) The Aldrich Library of ${ }^{13} \mathrm{C}$ and ${ }^{1} \mathrm{H}$ FT-NMR Spectra, Wiley, New York.

(26) Smith, M. (2010) Organic Chemistry: An Acid-Base Approach, CRC Press, New York.

(27) Brigelius, R., Muckel, C., Akerboom, T. P. M., and Sies, H. (1983) Identification and quantitation of glutathione in hepatic protein mixed disulfides and its relationship to glutathione disulfide. Biochem. Pharmacol. 32, 2529-2534.

(28) Zaborina, O., Daubaras, D. L., Zago, A., Xun, L., Saido, K., Klem, T., Nikolic, D., and Chakrabarty, A. M. (1998) Novel pathway for conversion of chlorohydroxyquinol to maleylacetate in Burkholderia cepacia AC1100. J. Bacteriol. 180, 4667-4675. 